\title{
Radial Basis Function Aided Space-Time Equalization in Dispersive Fading Uplink Environments
}

\author{
A. Wolfgang, S. Chen, L. Hanzo \\ School of ECS, University of Southampton, SO17 1BJ, UK \\ Tel: +44-23-8059 3125, Fax: +44-23-8059 4508 \\ Email: \{aw03r.sqc,lh\}@ecs.soton.ac.uk, http://www-mobile.ecs.soton.ac.uk
}

\begin{abstract}
A novel Radial Basis Function Network (RBFN) assisted Decision-Feedback aided Space-Time Equalizer (DF-STE) designed for receivers employing multiple antennas is proposed. The Bit Error Rate (BER) performance of the RBFN aided DF-STE is evaluated when communicating over correlated Rayleigh fading channels, whose Channel Impulse Response (CIR) is estimated using a Kalman filtering based channel estimator. The proposed receiver structure outperforms the linear Minimum Mean-Squared Error benchmarker and it is less sensitive to both error propagation and channel estimation errors.
\end{abstract}

\section{INTRODUCTION}

The capability of receivers employing multiple antennas to increase the achievable system capacity and to suppress the effects of cochannel interference has motivated intense research in the field of space-time equalization [1]. Most contributions however focus on sub-optimal linear receivers or investigate the performance of Maximum-Likelihood Sequence Estimators (MLSE), which suffer from an exponentially increasing complexity as a function of the delay-spread encountered. Owing to encountering non-minimum phase channels the received signal constellation may become linearly non-separable and in order to counteract this problem we introduce a novel non-linear Radial Basis Function Network (RBFN) [2] assisted Space-Time Equalizer (STE) for synchronous uplink communication scenarios. For the sake of complexity reduction an RBF-aided decision feedback (DF) structure is introduced, which necessitates the joint detection of all users. The described receiver structure resembles the Code Division Multiple Access (CDMA) Multi-User Detector (MUD) derived in [3]. However, the DF structure presented in this paper yields a substantial reduction of the computational cost imposed by the joint detection of all users. The investigated scenario assumes the presence of multiple Mobile Stations (MSs) communicating with the Base-Station (BS) over Single-Input Multiple-Output (SIMO) correlated Rayleigh fading channels, which are generated employing a procedure based on the Third Generation Partnership Project's (3GPP) Spatial Channel Model (SCM) [4]. In oder to highlighten the effects of channel estimation errors, the CIR of all users is estimated jointly with the aid of a Kalman filter based channel estimator.

The remainder of the paper is organized as follows. In Section II we will present our system model, which is used in Section III to derive our receiver structure, namely the RBFN aided DF-STE in Section III-A and the Kalman filtering based channel estimator in Section III-B. In Section IV the channel model is introduced, which is

The financial support of the EU under the auspices of the Phoenix and Newcom projects is gratefully acknowledged. The authors are also grateful to their colleagues for the enlightenment gained within the Phoenix consortium. employed in Section V for characterizing the achievable performance of the receiver. In Section VI we offer our conclusions.

\section{SySTEM MODEL}

The system considered consists of $Q$ number of Binary Phase Shift Keying (BPSK) modulated sources and a BS receiver, which is assumed to employ $L$ antennas. The channel output signal of the $l^{\text {th }}$ antenna element at time instant $t$ can then be written as

$$
x_{l}(t)=\sum_{q=1}^{Q} \sum_{k=0}^{K} h_{l q, k} s_{q}(t-k)+\eta(t),
$$

where $h_{l q, k}$ is the complex valued channel gain of the $k^{\text {th }}$ multipath component describing the channel between the $q^{\text {th }}$ source and the $l^{\text {th }}$ receiver antenna, $K$ is the number of multi-path components and $\eta(t)$ is the complex valued Additive White Gaussian Noise (AWGN) having a variance of $E\left[\left|\eta_{l}(t)\right|^{2}\right]=2 \sigma^{2}$. Each of the receiver's antenna elements is followed by a tapped delay line of length $m$, which is also referred to as the feed-forward section of the RBF-aided STE, as shown in Figure 1. The channel's output can be expressed in vectorial notation by the super-vector $\mathbf{x}(t)=$ $\left[\boldsymbol{x}(t)^{T}, \ldots, \boldsymbol{x}(t-m+1)^{T}\right]^{T}$, where $\boldsymbol{x}(t)$ is a column vector hosting the $L$ number of antenna-element output signals $x_{l}(t)$ given in Equation (1). The relation between the signal transmitted by the $Q$ sources and the channel's output for channel tap $k$ is described by a $(L \times Q)$-dimensional matrix $\boldsymbol{H}_{k}$, where the $(l q)^{t h}$ element of the matrix is given as $h_{l q, k}$. The super-matrix $\mathbf{H}$ representing the total system can then be obtained by concatenating the $(L \times Q)$ dimensional matrices $\boldsymbol{H}_{k}$, yielding:

$$
\mathbf{H}=\left(\begin{array}{cccccc}
\boldsymbol{H}_{k} & \cdots & \boldsymbol{H}_{k-m+1} & 0 & \cdots & 0 \\
& & \ddots & & \ddots & \\
0 & \cdots & 0 & \boldsymbol{H}_{k} & \cdots & \boldsymbol{H}_{k-m+1}
\end{array}\right) .
$$

The channel's output vector $\mathbf{x}(t)$ can now be expressed as

$$
\begin{aligned}
\mathbf{x}(t)= & \mathbf{H}\left[\boldsymbol{s}(t)^{T}, \ldots, \boldsymbol{s}(t-m+1)^{T}\right]^{T} \\
& +\left[\boldsymbol{\eta}_{1}(t)^{T}, \ldots, \boldsymbol{\eta}_{L}(t)^{T}\right]^{T} \\
= & \mathbf{H s}(t)+\boldsymbol{\eta}(t) \\
= & \overline{\mathbf{x}}(t)+\boldsymbol{\eta}(t),
\end{aligned}
$$

where $s(t)=\left[s_{1}(t), \ldots, s_{Q}(t)\right]^{T}$ is a column vector containing the symbols transmitted by the $Q$ sources and $\eta_{l}(t)=$ $\left[\eta_{1}(t), \ldots \eta_{l}(t-m+1)\right]^{T}$. Assuming that all sources transmit with identical power, the Signal to Noise Ratio (SNR) of user $q$ is given 
as

$$
\mathrm{SNR}_{q}=\frac{\sum_{l=1}^{L} \sum_{k=0}^{K} E\left[\left|h_{l q, k}\right|^{2}\right]}{2 \sigma^{2} L} .
$$

\section{RECEIVER}

The proposed receiver structure consists of two parts, namely the RBFN assisted DF-STE and a Kalman filter based channel estimator. The RBFN parameters may be estimated directly, but this approach has the drawback that it requires a long training period and has a poor tracking performance. By contrast, the cost function that is more straightforward to minimize for the channel estimator is the MMSE and the RBFN may be constructed based on the MMSE-optimized channel estimator, as it will be shown in the next sub-section.

\section{A. Decision Feedback Aided STE}

The performance of both linear and non-linear equalizers can be enhanced by incorporating a decision feedback structure in the receiver [2], as shown in Figure 1. In addition to the feed-forward section, the DF-STE is then characterized by the decision delay $\Delta$ and the decision feedback order $n$. Note that the oldest symbol vector, which still influences the detected symbol $\hat{s}_{q}(t-\Delta)$ is $s(t-m+1-K)$. Furthermore, the oldest feedback symbol vector is $\boldsymbol{s}(k-\Delta-n)$. Without loss of generality we therefore chose $n=m+K-1-\Delta$ for the derivation of the proposed DF-STE. In order to describe the feedback structure, we first divide the system matrix $\mathbf{H}$ into two sub-matrices $\mathbf{H}=\left[\begin{array}{ll}\mathbf{H}_{1} & \mathbf{H}_{2}\end{array}\right]$, where $\mathbf{H}_{1}$ hosts the first $Q(\Delta+1)$ columns of $\mathbf{H}$ and $\mathbf{H}_{2}$ represents the last $Q n$ columns in $\mathbf{H}$. The array output can then be written as

$$
\mathbf{x}(t)=\mathbf{H}_{1} \mathbf{s}_{1}(t)+\mathbf{H}_{2} \mathbf{s}_{2}(t)+\boldsymbol{\eta}(t),
$$

where $\mathbf{s}_{1}(t)=\left[s(t)^{T} \ldots s(t-\Delta)^{T}\right]^{T}$ indicates the symbols in the feed-forward shift register and $\mathbf{s}_{2}(t)=$ $\left[\boldsymbol{s}(t-\Delta-1)^{T} \ldots s(t-\Delta-n)^{T}\right]^{T}$ denotes the symbols in the feedback register. Under the assumption that the feedback vector is correct. Equation (4) can be re-written as

$$
\mathbf{r}(t)=\mathbf{x}(t)-\mathbf{H}_{2} \tilde{\mathbf{s}}_{2}(t)=\mathbf{H}_{1} \mathbf{s}_{1}(t)+\boldsymbol{\eta}(t),
$$

where $\mathbf{r}(t)$ is the observation space owing to the decision feedback. For a given feedback vector the possible noise-free channel output states in this new observation space $\overline{\mathbf{r}}(t)$ may assume $n_{s}=$ $2^{Q(\Delta+1)}$ different values, depending on the transmitted symbol vector $\mathbf{s}^{(i)}, 1 \leq i \leq n_{s}$, yielding $\overline{\mathbf{r}}^{(i)}=\mathbf{H}_{1} \mathbf{s}_{1}^{(i)}$. The set of all possible desired output states in the translated space $\overline{\mathbf{r}}(t)$ can be partitioned into two subsets $\mathcal{R}_{q}^{ \pm}$, depending on the binary value of the transmitted symbol $s_{q}^{(i)}(t-\Delta)$ of the desired user $q$ as

$$
\mathcal{R}_{q}^{ \pm}=\left\{\overline{\mathbf{r}}_{q}^{(i, \pm)}=\mathbf{H}_{1} \mathbf{s}_{1}^{(i)} \text { if } s_{q}^{(i)}(t-\Delta)= \pm 1\right\} .
$$

Based on the space translation given in Equation (5), the decision function of the filter depicted in Figure 1 can be written as

$$
\begin{aligned}
\hat{s}_{q}(t-\Delta) & =\operatorname{sgn}\left(f_{B, q}(\mathbf{r})\right) \\
& =\left\{\begin{array}{ll}
+1 & \text { if } f_{B, q}(\mathbf{r}(t)) \geq 0 \\
-1 & \text { if } f_{B, q}(\mathbf{r}(t))<0
\end{array},\right.
\end{aligned}
$$

where the optimal Bayesian decision function [2] $f_{B, q}(\cdot)$ based on the difference of the associated conditional density functions is given

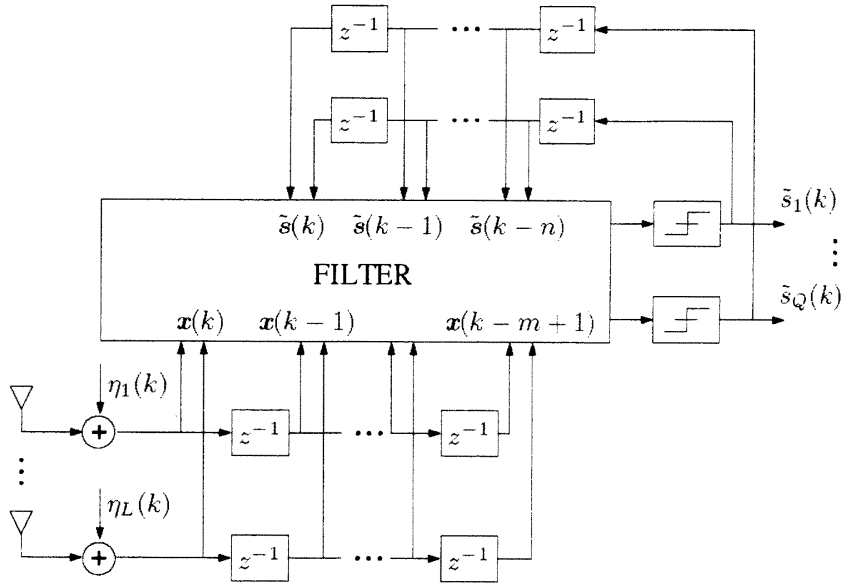

Fig. 1. General structure of a decision feedback aided space-time equalizer employing $L$ receive antennas in conjunction with a feed-forward order $m$ and feedback order $n$. The signals $s_{q}(k)$ of all $Q$ users are detected.

as

$$
\begin{aligned}
f_{B, q}(\mathbf{r}(k))= & P\left(\mathbf{x}(t) \mid s_{q}(t-\Delta)=+1\right) \\
& -P\left(\mathbf{x}(t) \mid s_{q}(t-\Delta)=-1\right) \\
= & \sum_{\overline{\mathbf{r}}_{q}^{(i,+)} \in \mathcal{R}_{q}^{+}} p\left(\mathbf{r}(t)-\overline{\mathbf{r}}_{q}^{(i,+)}\right) \\
& -\sum_{\overline{\mathbf{r}}_{q}^{(i,-)} \in \mathcal{R}_{q}^{-}} p\left(\mathbf{r}(t)-\overline{\mathbf{r}}_{q}^{(i,-)}\right) \\
= & \sum_{\overline{\mathbf{r}}_{q}^{(i,+)} \in \mathcal{R}_{q}^{+}} \alpha^{(i,+)} \exp \left(-\frac{\left\|\mathbf{r}(t)-\overline{\mathbf{r}}_{q}^{(i,+)}\right\|^{2}}{2 \sigma^{2}}\right) \\
& -\sum_{\overline{\mathbf{r}}_{q}^{(i,-)} \in \mathcal{R}_{q}^{-}} \alpha^{(i,-)} \exp \left(-\frac{\left\|\mathbf{r}(t)-\overline{\mathbf{r}}_{q}^{(i,-)}\right\|^{2}}{2 \sigma^{2}}\right),
\end{aligned}
$$

where $\overline{\mathbf{x}}_{i}^{q, \pm} \in \mathcal{R}^{q, \pm}, \alpha^{(i, \pm)}=\frac{p^{(i, \pm)}}{\left(2 \pi \sigma_{n}^{2}\right)^{2 m}}$. with the a-priori probabilities $p^{(i, \pm)}$ of $\mathbf{r}_{i}^{(q, \pm)}$.

The Bayesian DF-STE can be realized using a RBFN employing a Gaussian kernel. The response of such a RBFN is given as

$$
f_{R B F}(\mathbf{r}(k))=\sum_{i=1}^{N_{c}} w_{i} \phi\left(\mathbf{r}(k), \mathbf{c}^{(i)}\right)
$$

with

$$
\phi\left(\mathbf{r}(k), \mathbf{c}^{(i)}\right)=\exp \left(-\frac{\left\|\mathbf{r}(k)-\mathbf{c}^{(i)}\right\|^{2}}{\rho}\right),
$$

where the RBF centers $\mathrm{c}^{(i)}$ are set to the possible noise-free channel output states determined by the Channel Impulse Response (CIR), the radius $\rho$ is chosen to be $2 \sigma^{2}$ and the weights $w_{i}$ are set to +1 , if we have $\mathrm{c}^{(i)} \in \mathcal{R}^{q,+}$ and to -1 if $\mathrm{c}^{(i)} \in \mathcal{R}^{q,-}$. For the detection of $\tilde{s}_{q}(t-\Delta)$ the received signal vector $\mathbf{x}(t)$ is transformed into the translated space $\mathbf{r}(k)$ by subtracting the product of the feedback sequence $\tilde{\mathbf{s}}_{2}(t)$ and $\mathbf{H}_{2}$, given in Equation (5). In the translated space the signal is detected using the RBFN given in Equation (8).

The equations derived for the RBFN assisted DF-STE resemble the formulae published by Poor [3] in the context of CDMA MUD. However, there are two major differences. Firstly, the equations presented in [3] have been derived for a single-antenna CDMA MUD 
aided receiver and secondly, no decision feedback was invoked in the receiver. The introduction of decision feedback however has a beneficial effect on the computational cost imposed by the detector. Without decision feedback the computational complexity is proportional to $O\left(2^{Q(K+m)}\right)$, whereas for the decision feedback aided receiver it only increases with $O\left(2^{Q(\Delta+1)}\right)$. Considering a channel exhibiting a long CIR having only a short precursor and a long post-cursor, which allows us to opt for a moderate-length decision delay compared to the total length of the CIR, the employment of a decision feedback structure yields a significant complexity reduction.

The proposed DF aided receiver also has a lower complexity compared to other conventional trellis based receivers, such as for example the Viterbi STE, which has $2^{Q K}$ number of states for BPSK modulated MSs. The proposed receiver structure considers only $2^{Q(\Delta+1)}$ states, which might be significantly lower, depending on the pre-cursor duration of the CIR determining the decision delay $\Delta$ of the DF-STE. In addition to the reduced number of states the proposed scheme has the benefit of a short decision delay, which allows us to conveniently combine the DF-STE with a decision-directed channel estimator invoked for tracking time-varying channels.

The apparent inability of trellis based receiver structures to track time-variant channels in non-iterative receivers has therefore motivated the employment of a DF-MMSE STE similar to that proposed in [1] as a benchmarker for our advocated RBFN aided DF-STE. It has to be noted that the MMSE based receiver has a lower computational complexity, than either the trellis-based scheme or the RBFN assisted DF-STE.

\section{B. Kalman Estimator}

The Kalman filter based channel estimator employed was originally proposed in [5]. For the sake of deriving the estimator, the channel output vector of Equation (2) is re-written as

$$
\begin{aligned}
\boldsymbol{x}(t) & =\mathbf{S}(t)\left[\operatorname{vec}\left\{\boldsymbol{H}_{0}(t)\right\} \ldots \operatorname{vec}\left\{\boldsymbol{H}_{K}(t)\right\}\right]^{T} \\
& =\mathbf{S}(t) \mathbf{h}_{v e c} \\
& =\left(\left[\boldsymbol{s}(t)^{T} \ldots \boldsymbol{s}(t-K)^{T}\right] \otimes \mathbf{I}_{\mathrm{L}}\right) \mathbf{h}_{v e c}
\end{aligned}
$$

where $\otimes$ is the Kronecker product, $\mathbf{I}_{\mathrm{L}}$ is the $(L \times L)$-dimensional identity matrix and vec \{\} is the column-wise vector operator. Based on this formulation, the time-domain evolution of the vector channel $\mathbf{h}_{v e c}$, may be expressed using an Auto-Regressive (AR) model of order $p$, as follows:

$$
\mathbf{h}_{v e c}(t)=\sum_{i=1}^{p} \mathbf{A}(p) \mathbf{h}_{v e c}(t-i)+\mathbf{G}_{0} \mathbf{w},
$$

where $\mathbf{w}$ is a zero-mean i.i.d circular complex Gaussian vector process. The entries of the diagonal matrices $\mathbf{A}(l)$ and $\mathbf{G}_{0}$ can be calculated using the Yule-Walker method [6] in order to match the autocorrelation of the p-th oder AR model and the true auto-correlation of the channel. The parameters required for the construction of the estimator are the Doppler frequencies of the users and the delays associated with the different taps of the CIR. From the above model the Kalman filter equations can be constructed as proposed in [5].

The combination of the RBFN assisted DF-STE and the channel estimator works as follows. After a training period, when the channel estimator switches to a decision directed mode, the channel estimator predicts the channel coefficients involved in the construction of the feed-forward channel matrix $\mathbf{H}_{1}$ based on the previous channel estimates and updates this estimate when the associated symbols have been decided upon by the STE.

\section{Channel Model}

The channel model is based on the 3GPP-SCM described in [4] for system-level simulations.

Under the assumption of the underlying channel model [4] each CIR tap $\tilde{k}$ is associated with a cluster of scatterers $\mathcal{C}_{\tilde{k}}$ of the physical propagation environment which results in a cluster of propagation paths. These path result in a single, faded CIR tap since they are added up according to their phase. Each scattering cluster is characterized by two sets of parameters.

The first set of parameters is well known from other channel models and consists of the path delay and the power associated with the given cluster. The path delay is denoted by $\tau_{\tilde{k}}$ and the associated channel gain is $P_{\hat{k}}$, which may be expressed as $P_{\tilde{k}}=$ $E\left[\tilde{h}_{l q, \tilde{k}}(t) \tilde{h}_{l q, \tilde{k}}(t)^{*}\right]$, where the tilde indicates that the delays associated with the different cluster/CIR-taps are not necessarily symbol spaced. In [4] clear procedures have been proposed for determining the 'cluster-powers' and 'cluster-delays' for specific propagation environments.

In addition to the path powers and delays, spatial channel models are characterized by a set of geometric data determining the amount of correlation between the fading experienced by the signals arriving from a specific cluster at the different antenna array elements. These parameters are the angular spread $\sigma_{P A S}^{2}$ of the Power Azimuth Spectrum (PAS) and the angle $\theta_{\tilde{k}}$, in which the clusters are located with respect to the perpendicular of the antenna array. Given these parameters plus the antenna spacing $d$ spanning $\frac{\lambda}{2}, 4 \lambda$ or $10 \lambda$ at the BS antenna array the spatial covariance, which determines the correlation between the fading associated with different clusters can be generated using the approximate method proposed in [7].

The properties of the CIRs are best explained by considering the single tap of the PDP at index $\tilde{k}$ associated with user $q$, which may be written as $\tilde{\boldsymbol{h}}_{q, \tilde{k}}=\left[\tilde{h}_{1 q, \tilde{k}} \ldots \tilde{h}_{L q, \tilde{k}}\right]$. Assume now we have a correlated Rayleigh fading channel model, then CIR may be written as

$$
\tilde{\boldsymbol{h}}_{q, \tilde{k}}(t)=\sqrt{P_{\tilde{k}}} \mathbf{R}_{q, \tilde{k}}^{\frac{1}{2}} \mathbf{g}_{q, \tilde{k}}(t)
$$

where $\mathbf{g}_{q, \tilde{k}}$ is a. vector of complex Gaussian coefficients having zero mean and unity variance, $\mathbf{R}_{q, \tilde{k}}$ represents the spatial covariance matrices at the $\mathrm{BS}$ receiver.

The remaining question is, how to determine the cluster parameters. In the 3GPP-SCM [4], a number of clear strategies have been defined concerning how to generate the cluster parameters for given environments. For the urban micro-cellular environment, for example, six clusters are created corresponding to six channel taps, where the angular spread is fixed to $5^{\circ}$ and the cluster angles are drawn from a uniform distribution $\delta$ spanning the range of $\left(-40^{\circ}, 40^{\circ}\right)$. The propagation delay $\tau_{n}$ associated with a cluster is drawn from a uniform distribution spanning the range $(0 \mu s, 1.2 \mu s)$. Then the lowest of the six delays generated is subtracted from the others, hence the first delay is always zero. The power associated with a cluster can now be calculated as

$$
P_{\tilde{k}}=\frac{P_{\tilde{k}}^{\prime}}{\sum_{j=1}^{6} P_{\tilde{k}}^{\prime}} \text { with } P_{\tilde{k}}^{\prime}=10^{-\left(\tau_{\tilde{k}}-z_{\tilde{k}}\right) / 10},
$$

where $z_{\tilde{k}}$ are i.i.d. zero mean Gaussian random variables having a standard deviation of $3 \mathrm{~dB}$. For other specific environments the tech- 
nique becomes somewhat more elaborate. but nonetheless, follows the same principle.

The procedure of generating a channel matrix associated with correlated fading may be summarized as follows:

1) Generate one cluster for each of the $Q \tilde{K}$ number of CIR taps according to the procedure outlined in the 3GPP-SCM [4].

2) Calculate Mean Power and Delay of the CIR tap associated with the given cluster

3) Calculate the $Q \tilde{K}$ number of correlation matrices as proposed in [7].

4) Correlate the uncorrelated fading coefficients $\mathbf{g}_{q, \tilde{k}}(t)$ using Equation (12).

The CIR created with the aid of the above-mentioned procedure is fractionally spaced. The DF-STE however requires a symbolspaced CIR for its construction. Let us introduce the notation $\boldsymbol{h}_{l q}=$ $\left[h_{l q, 0} \ldots h_{l q, K}\right]^{T}$ and $\tilde{\boldsymbol{h}}_{l q}=\left[\tilde{h}_{l q, 0} \ldots \tilde{h}_{l q, \tilde{K}}\right]^{T}$, then the symbolspaced CIR is simply obtained with the aid of the following operation $\boldsymbol{h}_{l q}=\mathbf{P} \tilde{\boldsymbol{h}}_{l q}$, where the $(K \times \tilde{K})$-dimensional pulse-shaping matrix $\mathbf{P}$ is defined as

$$
\mathbf{P}=\left(\begin{array}{ccc}
g\left(-\tau_{1}\right) & \cdots & g\left(-\tau_{\tilde{K}}\right) \\
g\left(T_{s}-\tau_{1}\right) & \cdots & g\left(T_{s}-\tau_{\tilde{K}}\right) \\
\vdots & & \vdots \\
g\left(K T_{s}-\tau_{1}\right) & \cdots & g\left(K T_{s}-\tau_{\tilde{K}}\right)
\end{array}\right),
$$

with $g(t)$ being the impulse response of the plus-shaping filter. Note that the channel estimator only has to track the $\tilde{K}$ number of fractionally spaced channel taps. This may be readily achieved by replacing $\mathbf{S}(t)$ with $\mathbf{S}(t)\left(\mathbf{P} \otimes \mathbf{I}_{L Q}\right)$, where $\mathbf{I}_{L Q}$ is the $(L Q \times L Q)$ dimensional identity matrix.

\section{REsults}

Our initial study was carried out for two different types of Non-Line-Of-Sight (NLOS) environments, namely the micro-cellular urban and the macro-cellular urban environment [4]. The most salient simulation parameters are summarized in Table I. Note that the decision delays $\Delta$ chosen for the two different environments are significantly shorter than the length $K$ of the symbol spaced CIR considered. The system was assumed to support multiple MSs, having an identical average received signal power at the BS, which was employing a two-element array in all our simulations. The normalized Doppler frequency of all users was chosen to be $f_{D}=0.0003$. When the channel estimator was activated by the system studied, it used 150 training symbols and tracked the channel over the 600 payload symbols in a decision directed mode.

In Figure 2 our simulation results are shown for both the microcellular and the macro-cellular urban, environment using an interelement spacing of $d=4 \lambda$ and $d=\frac{\lambda}{2}$ at the BS. The graphs associated with the overloaded micro-cellular environment indicated as Cell Type (CT) 1, where the system was assumed to support three MSs, show that the MMSE DF-STE was incapable of detecting the different transmitters for both of the two different inter-element spacing scenarios. By contrast, the RBFN based detector is capable of detecting the users even for an antenna spacing of $d=\frac{\lambda}{2}$. The graphs associated with the macro-urban cellular environment supporting two MSs confirm the substantial performance difference between the RBFN aided and the MMSE based DF-STE. It may be concluded from Figure 2 that especially in hostile conditions,

\begin{tabular}{|l|c|c|}
\hline & Urban Micro (ACT1) & Urban Macro (CT2) \\
\hline \hline$g(t)$ & Raised Cosine & Raised Cosine \\
\hline$\tilde{K}$ & 6 & 6 \\
\hline$K$ & 8 & 14 \\
\hline$\sigma_{P A S}$ & $5^{\circ}$ & $2^{\circ}$ \\
\hline AoA & Uniform & $\mathcal{N}\left(0, \sigma_{A o A}\right)$ \\
& $\delta\left(-40^{\circ}, 40^{\circ}\right)$ & \\
\hline \hline$m$ & 2 & 4 \\
\hline$\Delta$ & 1 & 3 \\
\hline$L$ & 2 & 2 \\
\hline
\end{tabular}

TABLE I

SIMULATION PARAMETERS FOR THE GENERATION OF THE CORRELATION MATRICES BASED ON THE 3GPP-SCM AND FOR THE DF-STE.

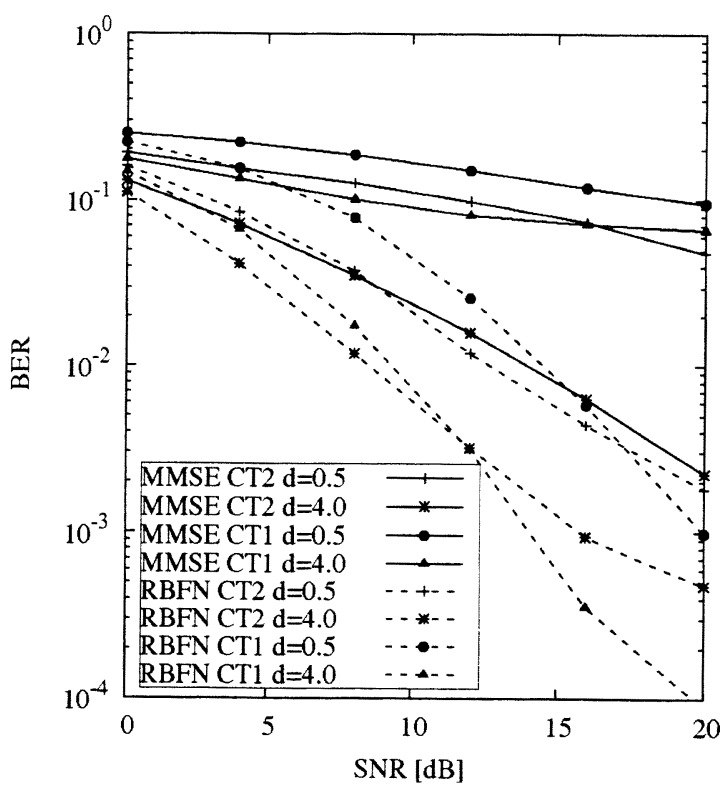

Fig. 2. Average BER of all MSs versus the SNR for different communication environments and different antenna spacings. In the micro-cellular urban environment (CT1) the number of MSs was chosen to be $Q=3$ and in the macro-cellular urban environment (CT2) it was set to $Q=2$. A normalized Doppler frequency of $f_{d}=0.0003$ was used for all MSs and it was assumed that the different MSs' signals were received at an equal average power. The channel was assumed to be known by the receiver. The remaining simulation parameters are summarized in Table I.

associated with either a small inter-element spacing or an overloaded scenario, the MMSE receiver falters, whereas the RBFN assisted receiver is capable of combating these effects.

The effects of estimated, rather than perfect Channel State Information (CSI) are shown in Figure 3 for the micro-cellular urban environment in the context of a system supporting two MSs and a BS using an inter-element spacing of $d=4 \lambda$. When comparing the MMSE-based receiver and the RBFN based receiver, both benefiting from perfect channel knowledge, it can be observed that the RBFNbased receiver outperforms its linear counterpart even in this nonoverloaded scenario. Considering the effect of imperfect channel estimation, it can be seen that the first-order Kalman filter associated with "p1" in Figure 3 exhibits tracking problems due to the mismatch of the AR(1) model and the true Rayleigh fading channel. By contrast, the second-order Kalman filter labelled as "p2" in Figure 3 


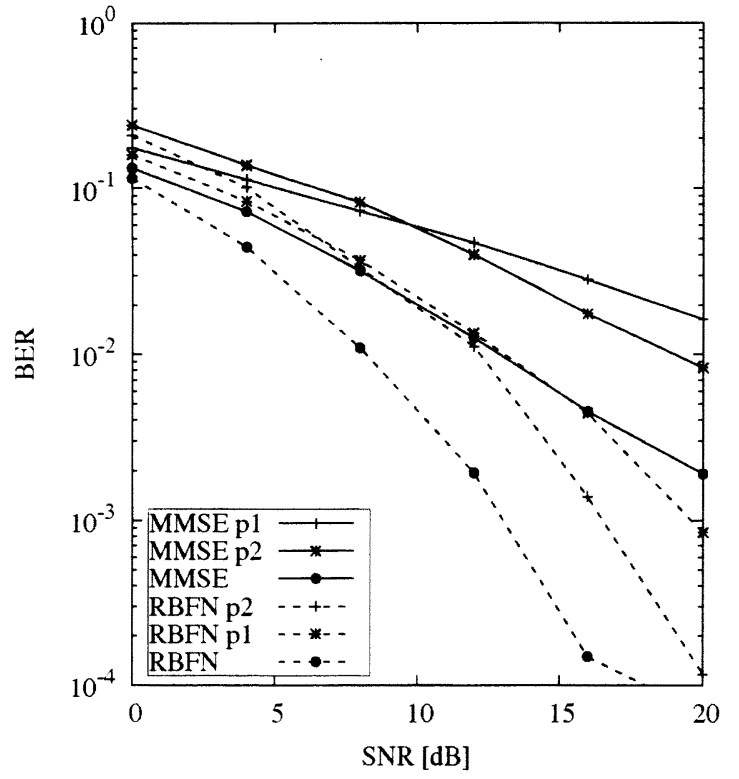

Fig. 3. Average BER of all MSs versus the SNR when communicating in a micro-cellular urban environment. A normalized Doppler frequency of $f_{d}=0.0003$ was employed for all users and it was assumed that all MSs' signals were received at an equal average power. The inter-element spacing was set to $d=4 \lambda$ and the number of MSs was chosen to be $Q=2$. Where the order $p$ of the channel estimator is not specified, perfect CIR knowledge was assumed. The remaining simulation parameters are summarized in Table I.

is capable of perfectly tracking the channel and is less affected by error propagation.

In Figure $4^{1}$ the BER performance recorded for perfect channel knowledge is shown as a function of the inter-element spacing $d$ for the micro-cellular urban environment, when the MSs are communicating with the $\mathrm{BS}$ at $\mathrm{SNR}=15 \mathrm{~dB}$. It can be seen that for two MSs the RBFN aided detector exhibits an error floor, which is caused by the ISI and the MAI encountered. As the number of users increases to three and four. the RBFN aided DF-STE becomes more capable of exploiting the diversity provided by the channel, when detecting the different users' signals. Interpreting this phenomenon further, increasing the inter-element spacing results in increasing the diversity gain of the system and hence more MSs can be supported by the system. For example, as seen in Figure 4 , in order to achieve a BER of $3 \cdot 10^{-3}$ an antenna spacing of $0.8 \lambda$ is required for two MSs, which is increased to $1.2 \lambda$ for three MSs and $1.6 \lambda$ for four MSs. However, in order to be able to exploit the extra spatial diversity at the receiver side, the detector first has to be capable of combating the ISI encountered. Note that since the micro-cellular urban environment is a rich scattering environment, the fading of the signals arriving at the different array elements is already rather uncorrelated for a relatively low antenna element separation and hence further increasing the distance between the receive antenna elements does not yield any significant diversity gain improvements.

\footnotetext{
${ }^{1}$ Also an inter-element spacing of $d=0$ does not make sense from a physical point of perspective, it is included in the results representing totally correlated fading associated with the different array elements.
}

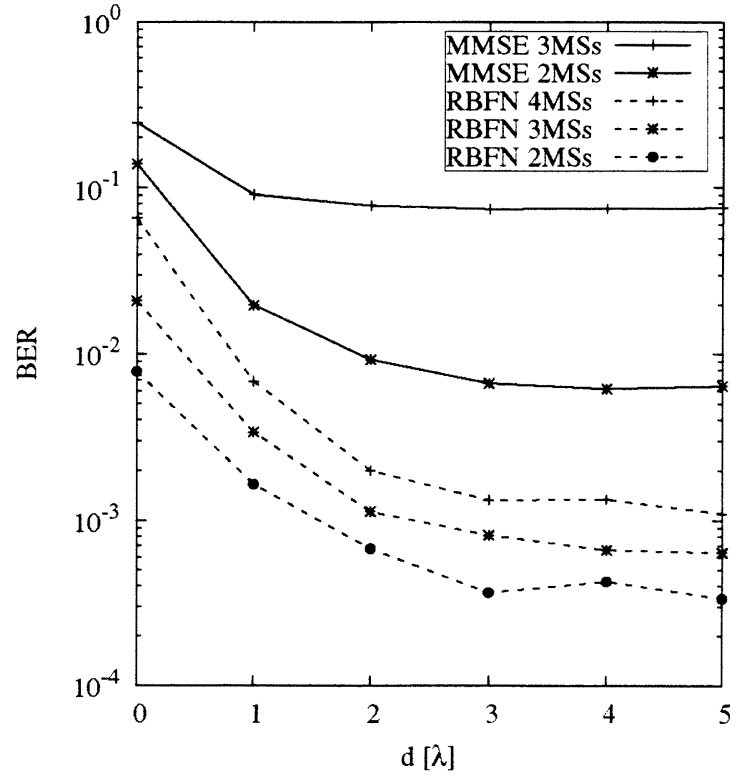

Fig. 4. Average BER of all MSs versus the antenna spacing, when communicating in a micro-cellular urban environment at $\mathrm{SNR}=15 \mathrm{~dB}$ for different number of MSs indicated as " $Q$ MSs". A normalized Doppler frequency of $f_{d}=0.0003$ was used for all MSs and it was assumed that the MSs' signals were received at an equal average power. The channel was assumed to be known by the receiver. The remaining simulation parameters are summarized in Table I.

\section{CONCLUSIONS AND FUTURE WORK}

Our simulation results have shown that the non-linear DF-STE outperforms the classic linear benchmarkers in the context of systems supporting at least the same number of users as the number of antenna elements, especially in hostile conditions inflicted either by a high MAI or poor spatial diversity imposed by a low inter-element spacing. It has also been shown that the RBFN aided DF-STE is more robust against both channel estimation errors and error-propagation. The relatively high complexity of both the detector and the channel estimator may be reduced by introducing an iterative structure, which is considered in our future work.

\section{REFERENCES}

[1] C. Tidestav, M. Sternad, A. Ahlen, "Reuse within a cell-interference rejection or multiuser detection?" IEEE Transactions on Communications, Vol.47, No.10, Oct. 1999, pp.1511-1522

[2] L. Hanzo, C. H. Wong, M. S. Yee: Adaptive Wireless Transceivers: Turbo-Coded, Turbo-Equalized and Space-Time Coded TDMA, CDMA, and OFDM Systems, John Wiley and IEEE Press, Feb. 2002

[3] X. Wang, H.V. Poor, "Iterative (turbo) soft interference cancellation and decoding for coded CDMA" IEEE Transactions on Communications, Vol.47, No.7, July. 1999, pp.1046-1061

[4] 3rd Generation Partnership Project (3GPP), "TR 25.996 V6.1.0, Spatial Channel Model for Multiple-Input Multiple-Output (MIMO) simulations", 2003

[5] C. Komninakis, C. Fragouli, A.H. Sayed, R.D. Wesel, "Multi-input multi-output fading channel tracking and equalization using Kalman estimation" IEEE Transactions on Signal Processing, Vol.50. No.5, May. 2002, pp.1065-1076

[6] A. Papoulis: Probability Random Variables and Stochastic Processes, 3rd ed. New York: McGraw-Hill, 1991

[7] A. Forenza, D.J. Love, R.W Heath Jr., "A low complexity algorithm to simulate the spatial covariance matrix for clustered MIMO channels" Proceedings of IEEE Vehicular Technology Conference, May 2004, CDROM 